\title{
The Role of Pattern Recognition in Computer-Aided Diagnosis and Computer-Aided Detection in Medical Imaging: A Clinical Validation
}

\author{
Srinivasan Nagaraj \\ Sr.Asst. Professor \\ Dept. of CSE, GMRIT \\ Rajam - 532127, AP, India
}

\author{
G Narasinga rao \\ Asst. Professor \\ Dept. of CSE, GMRIT \\ Rajam - 532127, AP, India
}

\author{
K Koteswararao \\ Asst. Professor \\ Dept. of CSE, GMRIT \\ Rajam - 532127, AP, India
}

\begin{abstract}
The idea to use texture analysis in medical imaging has been considered since the early 1970s. However, the exciting evolution of both texture analysis algorithms and computer technology revived researchers' interest in applications for medical imaging in recent years. Technical innovations in medical cross-sectional imaging have opened up new vistas for the exploration of the human body, enabling both high spatial and temporal resolution. On Difficulties Caused by Missing Reference Image Datasets One of the main problems in the development of systems for the analysis of medical images or segmentation algorithms is the set of images that is used for both the development and the testing of the system. This paper proposes a diagnostic scheme follows a familiar two-step approach. Initially, textural information is extracted from the image. Subsequently, the extracted information is fed into a decisional algorithm that is designed to perform the diagnostic task.The talk covers new methods for pattern recognition and computer-aided diagnosis in the field of MRI, such as functional MRI for human brain mapping, therapy control by automatic lesion detection in multiple sclerosis, and new approaches to breast cancer diagnosis in MRI mammography. In the light of such innovative techniques for pattern recognition in biomedicine, the increasing demand for publicly accessible validation platforms is emphasized. With image texture analysis in the role of the visual perceptional function, the process of feature extraction and image coding is achieved. The extracted features can now be merged into a diagnosis by using a decision-making algorithm, with choices that range from the rule-based models to the traditional statistical analysis to the more popular (and often more successful) artificial intelligence techniques, such as neural networks and genetic algorithms. By using image texture analysis as the preprocessing step in CAD schemes, the input generation process is automated and, therefore, is reproducible and robust. Some techniques represent texture on the basis of the spectral properties of an image. Others are model-based techniques that analyze texture by identifying an appropriate model that reflects the prior beliefs and knowledge about the type of images to be analyzed. There are textural features that describe local image statistics and others that describe global statistics.
\end{abstract}

\section{Keywords}

Textual image, CAD, Pattern recognition, Pattern matching.

\section{INTRODUCTION}

Pattern recognition is "the act of taking in raw data and taking an action based on the category of the pattern" ${ }^{[1]}$. Most research in pattern recognition is about methods for supervised learning and unsupervised learning. Pattern recognition aims to classify data (patterns) based either on a priori knowledge or on statistical information extracted from the patterns. The patterns to be classified are usually groups of measurements or observations, defining points in an appropriate multidimensional space. This is in contrast to pattern matching, where the pattern is rigidly specified. A complete pattern recognition system consists of a sensor that gathers the observations to be classified or described, a feature extraction mechanism that computes numeric or symbolic information from the observations, and a classification or description scheme that does the actual job of classifying or describing observations, relying on the extracted features. The classification or description scheme is usually based on the availability of a set of patterns that have already been classified or described. This set of patterns is termed the training set, and the resulting learning strategy is characterized as supervised learning. Learning can also be unsupervised, in the sense that the system is not given an a priori labeling of patterns, instead it itself establishes the classes based on the intrinsic regularities of the patterns. The classification or description scheme usually uses one of the following approaches: statistical (or decision theoretic) or syntactic (or structural). Statistical pattern recognition is based on statistical characterizations of patterns, assuming that the patterns are generated by a probabilistic system. Syntactical (or structural) pattern recognition is based on the structural interrelationships of features. A wide range of algorithms can be applied for pattern recognition, from simple naive Bayes classifiers and neural networks to the powerful k-nearest neighbor algorithm decision rules. Pattern recognition is more complex when templates are used to generate variants. For example, in English, sentences often follow the "N-VP" (noun - verb phrase) pattern, but some knowledge of the English language is required to detect the pattern. Pattern recognition is studied in many fields, including psychology, ethology, cognitive science and computer science. Holographic associative memory is another type of pattern matching where a large set of learned patterns based on cognitive meta-weight is searched for a small set of target patterns.

\subsection{Uses}

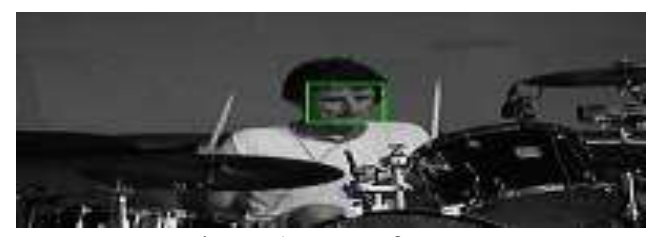

Figure 1. Face of Image 
The face was automatically detected by special software. Within medical science, pattern recognition is the basis for computer-aided diagnosis (CAD) systems. CAD describes a procedure that supports the doctor's interpretations and findings. Typical applications are automatic speech recognition, classification of text into several categories (e.g. spam/non-spam email messages), the automatic recognition of handwritten postal codes on postal envelopes, or the automatic recognition of images of human faces. The last two examples form the subtopic image analysis of pattern recognition that deals with digital images as input to pattern recognition systems. $^{[1][2]}$

\section{COMPUTER-AIDED DIAGNOSIS: ROLE OF IMAGE TEXTURE ANALYSIS}

The concept of 'computer aided diagnosis' (which is also called CAD) refers to software that analyses a radiographic finding to estimate the likelihood that the feature represents a specific disease process (e.g. benign versus malignant). To my knowledge, this technology has not yet been approved for clinical use. Currently, methods of image texture analysis are undergoing great development and utilization within the field of medical imaging. Given the general interest and striking growth in computer-aided diagnosis (CAD), the application of texture analysis in the diagnostic interpretation of radiologic images has become a rapidly expanding field of research. Proposed diagnostic scheme follows a familiar two-step approach. Initially, textural information is extracted from the image. Subsequently, the extracted information is fed into a decisional algorithm (eg. an artificial neural network) that is designed to perform the diagnostic task. However, from a methodological point of view, the main attraction of this study is that the combination of image texture analysis and automated decision making offers a promising approach to a clinical challenge (2). Successful applications of the above CAD strategy have been reported for other types of medical images (3-12). While further development and testing is required to establish the true clinical effect of such decisionsupport systems, texture analysis appears to open a new exciting path in the journey toward $\mathrm{CAD}$ in radiology. Consequently, two questions are raised: What is the realistic contribution of texture analysis in the computer-aided interpretation of medical images, and to what extent can it be expected to improve interpretative accuracy?

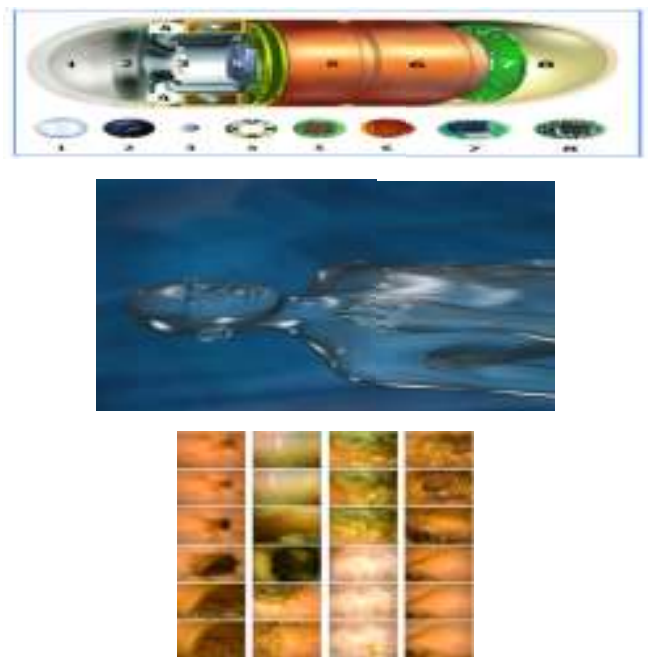

Figure 2. Image Textures

\subsection{Deconstructing the diagnostic Process}

The diagnostic interpretation of medical images is a multifaceted task. Its objective is the accurate detection and precise characterization of potential abnormalities - a crucial step toward the institution of effective treatment. Achieving this goal relies on the radiologists' successful integration of two distinct processes: $(a)$ the process of image perception to recognize unique image patterns and $(b)$ the process of reasoning to identify relationships between perceived patterns and possible diagnoses. Both processes depend heavily on the radiologists' empirical knowledge, memory, intuition, and diligence. Unquestionably, the radiologists approach the diagnostic task with a level of intelligence, flexibility, and common sense that is difficult to duplicate with a computer. Despite decades of intensive research in biologic visual systems and perceptual intelligence, there is a limited understanding and an ongoing debate about the basic mechanisms that underlie visual perception. In contrast, there is universal agreement that texture is a rich source of visual information and is a key component in image analysis and understanding in humans. Texture is known to provide clues about scenic depth and surface orientation and, as such, describes the content of both natural and artificial images. Also, there is evidence of perceptual learning in texture-coding mechanisms and in textural discrimination. In light of this, engineers have focused their attention on developing algorithms that can quantify the textural properties of an image. Empirical evidence and researchers' inventiveness have led to numerous algorithms for texture analysis. The available algorithms typically differ in the type of image information that is captured and in the coding mechanism. The evolution and diversity of available techniques for texture analysis are a testament to the advancement of this field. An extensive survey of textural definitions, models, and analytic algorithms can be found elsewhere. Texture analysis is ultimately concerned with automated methods that can derive image information from a purely computational point of view. As such, it is nothing more than another type of numeric manipulation of digital or digitized images to get quantitative measurements. But contrary to the discrimination of morphologic information (i.e., shape, size), there is evidence that the human visual system has difficulty in the discrimination of textural information that is related to higher-order statistics or spectral properties on an image. Consequently, texture analysis can potentially augment the visual skills of the radiologist by extracting image features that may be relevant to the diagnostic problem but that are not necessarily visually extractable. Nonetheless, texture analysis is not a panacea for the diagnostic interpretation of radiologic images. The pursuit of texture analysis is based on the hypothesis that the texture signature of an image is relevant to the diagnostic problem at hand. Furthermore, the effectiveness of texture analysis is bound by the type of algorithm that is used to extract meaningful features. A decision algorithm that uses automatically extracted features has a better chance of producing a robust CAD system. A CAD tool that uses automatically extracted features addresses an established clinical weakness of the diagnostic process and also complements the radiologists' perceptive abilities. Texture analysis is well suited to this problem since the radiologists themselves rely on visual texture to detect and describe breast lesions on US images. The fact that the image processing used in the study can be easily performed with existing software packages dramatically simplifies the incorporation of the proposed $\mathrm{CAD}$ tool into the clinic. The effectiveness of any 
CAD tool will always be conditional on two things: (a) how well the selected features describe the disease states that need to be discriminated and $(b)$ how well the study group reflects the overall target patient population for the CAD tool.

\section{CAD -CLINICAL IMPLEMENTATION}

Computer aided detection (CAD) is a technology designed to decrease observational oversights - and thus the false negative rates - of physicians interpreting medical images. Prospective clinical studies have demonstrated an increase in breast cancer detection with CAD assistance. This overview briefly describes the metrics that have been used to define CAD system performance. More recently, computer programs have been developed and approved for use in clinical practice that aid radiologists in detecting potential abnormalities on diagnostic radiology exams. This application has been termed computer aided (or assisted) detection, commonly referred to as CAD. The term 'computer-aided detection' refers to pattern recognition software that identifies suspicious features on the image and brings them to the attention of the radiologist, in order to decrease false negative readings. As currently used, the radiologist first reviews the exam, then activates the CAD software and re-evaluates the CAD-marked areas of concern before issuing the final report. CAD is currently FDA and CE approved for use with both film and digital mammography, for both screening and diagnostic exams; for chest CT; and, for chest radiographs. The primary goal of CAD is to increase the detection of disease by reducing the false negative rate due to observational oversights. The use of a computer rather than a second human observer has the advantage of not increasing the demands on the radiologist (or trained observer) pool. An important aspect of either approach is to increase disease detection without an undo impact on the recall and work up rates. Finally, in some applications CAD, with its associated automated software tools, has the potential to provide workflow efficiencies. CAD algorithms are developed to search for the same features that a radiologist looks for during case review. Thus, for breast cancer on mammograms, the CAD algorithms search for micro calcifications and masses (both speculated and non speculated, architectural distortions and asymmetries). On chest radiographs and CT scans, current CAD applications search for pulmonary densities that possess certain physical characteristics, e.g. sphericity, that might represent lung nodules. Not surprisingly, CAD algorithms will mark features that meet the algorithm requirements, but which do not represent findings that the radiologist considers to warrant further investigation, i.e. false CAD marks. Also, at times a true positive CAD mark, upon review by the radiologist, is dismissed as not warranting further investigation. In this instance, the false negative report would be the result of an interpretive-rather than a perceptionerror.

\subsection{CAD Methodology}

$\mathrm{CAD}$ is fundamentally based on highly complex pattern recognition. X-ray images are scanned for suspicious structures. Normally a few thousand images are required to optimize the algorithm. Digital image data are copied to a CAD server in a DICOM-format and are prepared and analyzed in several steps.

\subsubsection{Preprocessing for \\ - Reduction of artifacts (bugs in images) \\ - Image noise reduction}

- Leveling (harmonization) of image quality for clearing the image's different basic conditions e.g. different exposure parameter.

\subsubsection{Segmentation for}

- Differentiation of different structures in the image, e.g. heart, lung, ribcage, possible round lesions

- Matching with anatomic databank

\subsubsection{Structure/ROI (Region of Interest) Analyze}

Every detected region is analyzed individually for special characteristics:

- Compactness

- Form, size and location

- Reference to close-by structures / ROIs

- Average greylevel value analyze within a ROI

- Proportion of greylevels to border of the structure inside the ROI

\subsubsection{Evaluation / classification}

After the structure is analyzed, every ROI is evaluated individually (scoring) for the probability of a TP. Therefore the procedures are:

- Nearest-Neighbor Rule

- Minimum distance classifier

- Cascade Classifier

- Bayesian Classifier

- Multilayer perception

- $\quad$ Radial basis function network (RBF)

- SVM

If the detected structures have reached a certain threshold level, they are highlighted in the image for the radiologist. Depending on the CAD system these markings can be permanently or temporary saved. The latter's advantage is that only the markings which are approved by the radiologist are saved. False hits should not be saved, because an examination at a later date becomes more difficult then.

\subsection{CAD Implementation background evaluation}

The CAD algorithms require a digital data set of the image for analysis. If the image is acquired on $\mathrm{x}$-ray film, such as a filmscreen mammogram, the analog image must first be digitized. However, the CAD algorithms can directly analyze images acquired in digital format, such as with digital mammography (FFDM) and CT.

In current practice (and as required by the FDA), the exam should first be reviewed and interpreted in the usual fashion. Only then are the CAD marks displayed, following which the radiologist re-reviews those areas that are prompted by the CAD system. Two important principles must be adhered to:

- Current CAD systems do not mark all actionable findings. Therefore, the absence of a CAD mark on a finding the radiologist was concerned about on his/her pre CAD review must not deter further evaluation. 
- Current CAD systems generate many more false $\mathrm{CAD}$ marks than true CAD marks. Therefore, it is the responsibility of the radiologist to determine if a $\mathrm{CAD}$ mark warrants further evaluation.

A CAD system can be evaluated in several ways, which includes analysis of data generated in a laboratory or test setting, and by the impact of CAD on radiologist performance in an actual clinical practice setting. 'Stand alone' sensitivity and specificity, this information can be obtained by observing the performance of a CAD system on a set of 'truth' cases. Truth is generally established by histological verification of the presence (e.g. cancer) or absence (e.g. clinical follow-up) of disease. Sensitivity is determined by the percentage of positive cases in which the CAD system places a mark on the disease location. The number of false CAD marks per normal image or case is commonly used as a surrogate for specificity. The results of this exercise are, of course, dependent on the case collection. Bias, intended or not, in collecting positive cases that have more conspicuous findings will result in apparent superior CAD performance compared to cases that are less conspicuous, even though the $\mathrm{CAD}$ algorithm is the same. Thus, the same CAD algorithm will demonstrate varying sensitivities and specificities (false marks per case) depending on the case composition. A preferred method to compare CAD systems is to determine the sensitivity and false marker rates on the same set of 'truth' cases. These cases must be 'unknown' to the CAD system, that is, they should not have been used to train the CAD algorithms. Sufficient (and often large) numbers of cases will be needed in order to establish statistical significance of superiority or equivalence in performance when comparing CAD systems. 'Laboratory' studies of potential detection improvement These studies recruit radiologists (or other 'readers') to evaluate a set of 'truth' cases to determine the sensitivity and call back rate of the unaided reader (pre-CAD) with that of the reader with CAD assistance. Such studies are useful to assess the potential benefit of CAD and provide estimates of expected changes in disease detection and workup/recall rates. However, the test setting often compromises the performance of the reader, in that the readers may either over or under call the reviewed cases in a test environment. This can be done in a 'sequential read' clinical trial, in which the exam is first read prior to, and then following, CAD input [2-5]. The change in disease detection due to the CAD input, as well as the change in the recall/workup rates, will determine the contribution of CAD to patient management. Importantly, the percentage increase in disease detection should be concordant with, or less than, the percentage increase in the recall/workup rates.

\section{CONCLUSION}

The idea to use texture analysis in medical imaging has been considered since the early 1970s. However, the exciting evolution of both texture analysis algorithms and computer technology. On Difficulties Caused by Missing Reference Image Datasets One of the main problems in the development of systems for the analysis of medical images or segmentation algorithms is the set of images that is used for both the development and the testing of the system. This paper describes the method with image texture analysis in the role of the visual perceptional function, the process of feature extraction and image coding is achieved. The extracted features can now be merged into a diagnosis by using a decision-making algorithm, with choices that range from the rule-based models to the traditional statistical analysis to the more popular (and often more successful) artificial intelligence techniques, such as neural networks and genetic algorithms. By using image texture analysis as the preprocessing step in CAD schemes, the input generation process is automated and, therefore, is reproducible and robust. Thus far, study findings have shown that $\mathrm{CAD}$ can enhance the diagnostic performance of radiologists, if it is used as a second opinion. However, it is still difficult to predict its acceptance in medical diagnosis, and its acceptance will not be determined until findings from extensive clinical use demonstrate quantifiable benefits. At the same time, commercial CAD products will have to face new challenges related to marketing, technical support, modifications, and product life-cycles and practices in the health care environment are constantly redefined.

\section{REFERENCES}

[1] Chen DR, Chang RF, Huang YL. Computer-aided diagnosis applied to US of solid breast nodules by using neural networks. Radiology 1999; 213:407-412.

[2] D R. Brunelli, Template Matching Techniques in Computer Vision: Theory and Practice, Wiley, ISBN 978 0-470-51706-2, 2009 ([1] TM book).

[3] Fukunaga, Keinosuke (1990). Introduction to Statistical Pattern Recognition (2nd ed.). Boston: Academic Press. ISBN 0-12-269851-

[4] Bishop, Christopher (2006). Pattern Recognition and Machine Learning. Berlin: Springer. ISBN 0-387-310738 .

[5] Godfried T. Toussaint, ed (1988). Computational Morphology. Amsterdam: North-Holland Publishing Company.

[6] Kulikowski, Casimir A.; Weiss, Sholom M. (1991). Computer Systems That Learn: Classification and Prediction Methods from Statistics, Neural Nets, Machine Learning, and Expert Systems. Machine Learning. San Francisco: Morgan Kaufmann Publishers. ISBN 1-55860065-5.

[7] Krupinski EA. The future of image perception in radiology. Acad Radiol. 2003;10:1-3. [PubMed].

[8] Vittitoe NF, Baker JA, Floyd CE, Jr. Fractal texture analysis in computer-aided diagnosis of solitary pulmonary nodules. Acad Radiol 1997; 4:96-101.

[9] S Wei D, Chan HP, Helvie MA, et al. Classification of mass and normal breast tissue on digital mammograms: multiresolution texture analysis. Med Phys 1995; 22:1501-1513

[10] Chan HP, Sahiner B, Petrick N, et al. Computerized classification of malignant and benign microcalcifications on mammograms: texture analysis using an artificial neural network. Phys Med Biol 1997; 42:549-567.

[11] Petrick N, Chan HP, Wei D, Sahiner B, Helvie MA, Adler DD. Automated detection of breast masses on mammograms using adaptive contrast enhancement and texture classification. Med Phys 1996; 23:1685-1696.

[12] McPherson DD, Aylward PE, Knosp BM, et al. Ultrasound characterization of acute myocardial ischemia by quantitative texture analysis. Ultrason Imaging 1986; 8:227-240. 
[13] Ito M, Ohki M, Hayashi K, Yamada M, Uetani M, Nakamura T. Trabecular texture analysis of CT images in the relationship with spinal fracture. Radiology 1995; 194:55-59.

[14] Staff RT, Gemmell HG, Duff PM, et al. Decompression illness in sports divers detected with technetium-99mHMPAO SPECT and texture analysis. J Nucl Med 1996; 37:1154-1158.
[15] Lucht R, Brix G, Lorenz WJ. Texture analysis of differently reconstructed PET images. Phys Med Biol 1996; 41:2207-2219.

[16] Robinson PJ. Radiology's Achilles' heel: error and variation in the interpretation of the Roentgen image. $\mathrm{Br} \mathrm{J}$ Radiol 1997; 70:1085-1098.

[17] Anderson RE, Hill RB, Key CR. The sensitivity and specificity of clinical diagnostics during five decades: toward an understanding of necessary fallibility. JAMA $1989 ; 261: 1610-1617$ 